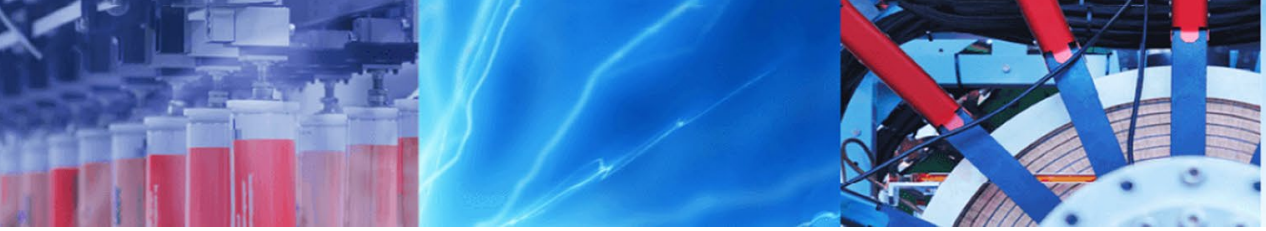

Research Article

\title{
Leapfrogging of electrical solitons in coupled nonlinear transmission lines: effect of an imperfect varactor
}

\author{
Nkongho Achere Akem ${ }^{1}$. Alain M. Dikandé ${ }^{1}$ D $\cdot$ B. Z. Essimbi ${ }^{3}$
}

Received: 14 October 2019 / Accepted: 20 November 2019 / Published online: 4 December 2019

(c) The Author(s) 2019 OPEN

\begin{abstract}
The leapfrogging dynamics of a pair of electrical solitons is investigated, by considering two capacitively coupled nonlinear transmission lines with and without intraline resistances. We discuss two distinct transmission line set-ups: in the first, we assume two RLC ladder lines with intraline varactors and a coupling linear capacitor, and in the second, we consider two capacitively coupled lossless lines with a varactor carrying impurity (imperfect diode) in one of the two interacting transmission lines. In the first context, we find that the soliton-pair leapfrogging mimics the motion of a damped harmonic oscillator, the frequency and damping coefficient of which are obtained analytically. Numerical simulations predict leapfrogging of the soliton pair when the differences in the initial values of the amplitude and phase are reasonably small, and the resistance is not too large. In the second context, leapfrogging occurs when the impurity rate is small enough and the differences in the initial values of the amplitude as well as phase are also small. As the impurity rate increases, the soliton signal in the imperfect line gets accelerated upon approaching the defective diode, causing only this specific soliton signal to move faster than its counterpart, leading to the suppression of leapfrogging.
\end{abstract}

Keywords Coupled nonlinear transmission lines · Soliton signals · Capacitive impurity · Adiabatic perturbation theory · Numerical simulations

\section{Introduction}

The Hirota circuit $[1,2]$ is a simple LC ladder circuit with a linear inductance, but an active feedback capacitor embedded within the main branch of the circuit. This circuit has long served as a paradigm for the generation and propagation of nonlinear signals in electrical networks, simulating the so-called Toda lattice $[3,4]$ and admitting exact soliton solutions [5-9]. In this electrical system, the nonlinearity balancing the dispersion (related to the ladder nature of the line) is introduced by a capacitor, whose capacitance is controlled by the imposed bias voltage, thus acting like a capacitive diode ("varicap" diode or varactor). The nonlinear signal generated in this nonlinear transmission line (NLTL) is a localized electrical signal with a bell shape, propagating with features of pulse soliton (i.e., translate at constant speed keeping a permanent bell shape) due to the effect of varactors periodically loaded throughout the line. NLTLs are of interest because of their applications in several fields, e.g., under large signal conditions NLTLs can serve as impulse compressors or frequency multipliers [10]. NLTLs have also proved to be of great practical use in extremely wideband focusing and shaping of signals [11]; in the microwave domain, they are ideal sources of highly stable large-amplitude sharp pulses [12].

Several studies have been devoted to modelling, both analytically and numerically, the propagation of nonlinear signals in NLTLs in various physical contexts

$\triangle$ Alain M. Dikandé, dikande.alain@ubuea.cm | 'Laboratory of Research on Advanced Materials and Nonlinear Science (LaRAMaNS), Department of Physics, Faculty of Sciences, University of Buea, PO Box 63, Buea, Cameroon. ${ }^{2}$ Present Address: Max Planck Institute for the Physics of Complex Systems (MPIPKS), Nöthnitzer Str. 38, 01187 Dresden, Germany. ${ }^{3}$ Laboratory of Electronics and Electrical Systems, Department of Physics, Faculty of Science, University of Yaoundé I, PO Box 812, Yaoundé, Cameroon. 
including resistive NLTLs, transmission lines with impurities, networks of coupled NLTLs and so on [13-27]. Two most common theoretical pictures have emerged, namely one in which the nonlinear electrical signals are soliton solutions to the Korteweg-de Vries (KdV) or coupled KdV equations [27-29] and one in which they are looked out as modulated envelope solitons described by the nonlinear Schrödinger or coupled nonlinear Schrödinger equations $[10,12,18,19]$. Much interesting to us, recent theoretical as well as numerical works $[30,31]$ have established that under specific conditions, the coupling of two NLTLs can promote novel interesting configurations of soliton bound states in which soliton pairs propagate with opposite phases, but nearly equal velocities. Bound soliton states of this kind, known as leapfrogging solitons, have actually been predicted and observed experimentally in many other physical contexts [32-38] as, for instance, in hydrodynamics and plasma dynamics.

In general, the amplitudes and phases of two leapfrogging pulses depend on their initial positions and initial velocities, such that as they propagate they remain always close one to another with their amplitude and phase differences vanishing periodically with time. In the specific context of coupled NLTLs, leapfrogging propagation of soliton pairs provides means to convey pairs of largeamplitude signals at low energy cost from their interactions. Indeed when the difference in velocities of the two signals is very small, their interaction is optimized, thus favouring a bound state in which the electrical energy will be alternately transferred from the leading soliton signal to the trailing soliton signal. This leads to a periodic change in positions of the two solitons, a leading one becoming a trailing soliton and vice versa. So to say, the leapfrogging motion can be used to manage the transmission of pairs of travelling electrical pulses in electrical networks, putting into play a minimum possible power loss from the individual electrical soliton signals.

In a previous study [31], we investigated the leapfrogging dynamics of soliton pairs propagating along two LC NLTLs, weakly coupled by a linear capacitance shunted with a linear resistance. We obtained that the inclusion of a resistive element in the shunt branch of the coupling capacitance enables to control the amplitude and phase differences of the interacting pulses during their propagation. Instructively, the coupled model considered in this previous study was an extension of the study done in ref. [29] where the author addressed the problem considering only the capacitive coupling. In the present work, we are interested in the leapfrogging motion of a soliton pair in two distinct physical contexts: first, we consider the case of two RLC NLTLs coupled via a linear capacitance and two capacitively coupled LC NLTLs one of which contains a defective varactor. We first derive, using Kirchhoff's voltage and current rules, the discrete set of nonlinear equations for the coupled NLTLs in the two physical contexts. Next, seeking for pulse signals, a multiple-scale expansion of solutions is applied in the full continuum limit which enables us to obtain a set of coupled KdV equations in the relevant scale. The coupled set of KdV equations is then treated analytically within the framework of the adiabatic perturbation theory [39], by defining appropriate variables for leapfrogging of the two KdV pulses as they propagate at nearly equal amplitudes and velocities. Their leapfrogging are explored numerically by means of a sixth-order Runge-Kutta scheme with fixed steps [40], and conditions for suppression of leapfrogging are determined.

\section{Analysis of leapfrogging for coupled RLC NLTLS}

\subsection{Model, line equations and coupled dissipative KdV equations}

Consider two NLTLs as depicted in Fig. 1, where each elementary section in the line consists of a linear inductor $L$ in parallel with a nonlinear capacitor of capacitance $C=C(V)$. The two lines are coupled by means of linear capacitor $C_{m}$ at each mode.

The nonlinear capacitors are varactor diodes, and except for their opposite polarities in our study, we shall use a common type of varactor diode with a Schottky barrier for both lines [30, 31]. Therefore, our Schottky varactors can be represented by the following capacitance-voltage characteristics [41]:

$$
\begin{aligned}
& C_{1}(x)=C_{0} /\left(1-\frac{x}{V_{\mathrm{J}}}\right)^{m}, \\
& C_{2}(x)=C_{0} /\left(1+\frac{x}{V_{\mathrm{J}}}\right)^{m},
\end{aligned}
$$

where $C_{0}, V_{\text {J }}$ and $m$ are, respectively, the zero-bias capacitance, the junction potential and the grading coefficient $[41,42]$. We assume that the bias voltages in lines 1 and 2 are $-V_{\mathrm{b}}$ and $V_{\mathrm{b}}$, respectively, reflecting the opposite polarities of varactor diodes loaded on the two lines. For convenience, we define:

$C_{\mathrm{b}} \equiv C_{0} /\left(1+\frac{V_{\mathrm{b}}}{V_{\mathrm{J}}}\right)^{m}$

an effective zero-bias capacitance which below turns out to be a relevant characteristic parameter.

Applying Kirchhoff's rules on the two coupled electrical ladder circuits, we obtain the following set of discrete transmission line equations: 
Fig. 1 Equivalent representation of two nonlinear RLC transmission lines with Schottky-type in-line varactors $C(V)$, coupled by linear capacitors $C_{m}$
$L \frac{\mathrm{d}}{\mathrm{d} t}\left(J_{n-1}-J n\right)+R\left(J_{n-1}-J_{n}\right)=W_{n-1}-2 W_{n}+W_{n+1}$,

$L \frac{\mathrm{d}}{\mathrm{d} t}\left(I_{n-1}-\ln \right)+R\left(I_{n-1}-I_{n}\right)=V_{n-1}-2 V_{n}+V_{n+1}$,

$J_{n-1}-J n=\frac{\mathrm{d} Q_{n}}{\mathrm{~d} t}+C_{m} \frac{\mathrm{d}}{\mathrm{d} t}\left(W_{n}-V_{n}\right)$

$I_{n-1}-\ln =\frac{\mathrm{d} q_{n}}{\mathrm{~d}} t+C_{m} \frac{\mathrm{d}}{\mathrm{d} t}\left(V_{n}-W_{n}\right)$.

In the above set, $W_{n}$ and $J_{n}$ are, respectively, the voltage and current of the $n$th section in line 1 , and $V_{n}$ and $I_{n}$ are, respectively, the voltage and current of the $n$th section in line 2 . In the continuum limit, when the size of elementary sections in the circuits is very small compared with the length of the transmission lines, the right-hand side of Eqs. (4) and (5) can readily be approximated with partial derivatives with respect to a continuum variable $x=n l$. This, more exactly, corresponds to the long-wavelength approximation which consists in Taylor expanding the discrete variables $W_{n \pm 1}$ and $V_{n \pm 1}$, i.e.,

$W_{n \pm 1}=V_{n} \pm \frac{\partial W}{\partial x}+\frac{1}{2} \frac{\partial^{2} W}{\partial x^{2}} \pm \frac{1}{6} \frac{\partial^{3} W}{\partial x^{3}}+\frac{1}{24} \frac{\partial^{4} W}{\partial x^{4}}+\cdots$,

$V_{n \pm 1}=V_{n} \pm \frac{\partial V}{\partial x}+\frac{1}{2} \frac{\partial^{2} V}{\partial x^{2}} \pm \frac{1}{6} \frac{\partial^{3} V}{\partial x^{3}}+\frac{1}{24} \frac{\partial^{4} V}{\partial x^{4}}+\cdots$
Also, from the definition $\mathrm{d} Q_{n}=C\left(W_{n}\right) \mathrm{d} W_{n}$ and $\mathrm{d} q_{n}=C\left(V_{n}\right) \mathrm{d} V_{n}$ and setting $\alpha_{0}=m / V_{\mathrm{J}}$, Eqs. (4)-(7) reduce to:

$$
\begin{aligned}
\mathrm{LC}_{0} & \left(\frac{\partial^{2} W}{\partial t^{2}}+\frac{\alpha_{0}}{2} \frac{\partial^{2} W^{2}}{\partial t^{2}}\right) \\
& +\mathrm{LC} C_{m} \frac{\partial^{2}}{\partial t^{2}}(W-V)+\mathrm{RC} \frac{\partial}{\partial t^{2}}(W-V) \\
& +\mathrm{RC}_{0}\left(\frac{\partial W}{\partial t}+\frac{\alpha_{0}}{2} \frac{\partial W^{2}}{\partial t}\right)=\frac{\partial^{2} W}{\partial x^{2}}+\frac{1}{12} \frac{\partial^{4} W}{\partial x^{4}}
\end{aligned}
$$

$$
\begin{aligned}
\mathrm{LC}_{0} & \left(\frac{\partial^{2} V}{\partial t^{2}}-\frac{\alpha_{0}}{2} \frac{\partial^{2} V^{2}}{\partial t^{2}}\right) \\
+ & \mathrm{LC}_{m} \frac{\partial^{2}}{\partial t^{2}}(V-W)+\mathrm{RC}_{m} \frac{\partial}{\partial t^{2}}(V-W) \\
& +\mathrm{RC}_{0}\left(\frac{\partial V}{\partial t}-\frac{\alpha_{0}}{2} \frac{\partial V^{2}}{\partial t}\right)=\frac{\partial^{2} V}{\partial x^{2}}+\frac{1}{12} \frac{\partial^{4} V}{\partial x^{4}}
\end{aligned}
$$

As we are interested in voltage signals with localized wave profile in space and time, it is useful to find appropriate equations reproducing such structures. In this goal, we choose the reductive perturbation method [43], in which the voltage variables $W$ and $V$ can be expanded in series according to:

$W(x, t)=-V_{\mathrm{b}}+\sum_{i=1}^{n} \epsilon^{i} W_{i}(x, t)$,

$V(x, t)=V_{\mathrm{b}}+\sum_{i=1}^{n} \epsilon^{i} V_{i}(x, t)$ 
In addition, we apply the following new transformations on the space and time coordinates, as well as on the resistance coefficient $R$ :

$z=\epsilon^{\frac{1}{2}}(x-\eta t), \tau=\epsilon^{\frac{3}{2}} t, R=\epsilon^{\frac{3}{2}} R_{1}$,

where $\eta=\left(\mathrm{LC}_{b}\right)^{-1 / 2}$ with $C_{\mathrm{b}}$ defined in (3). Furthermore, the coupling capacitance $C_{m}$ must be of order $\epsilon$ [31], i.e.,we should define $C_{m}=\epsilon C$. Substituting Eqs. (12) to (14) into Eqs. (10) and (11) and using the above transformation of the coupling capacitance, we obtain the following equations to the order $O\left(\epsilon^{3}\right)$ :

$$
\begin{aligned}
& -\frac{2}{\eta} \frac{\partial^{2} W_{1}}{\partial z \partial \tau}+\frac{\alpha_{0} C_{0}}{2 C_{\mathrm{b}}} \frac{\partial^{2} W_{1}^{2}}{\partial z^{2}}+\frac{C}{C_{\mathrm{b}}} \frac{\partial^{2}}{\partial z^{2}}\left(W_{1}-V_{1}\right) \\
& -R_{1} C_{\mathrm{b}} \eta \frac{\partial W_{1}}{\partial z}=\frac{1}{12} \frac{\partial^{4} W_{1}}{\partial z^{4}} \\
& -\frac{2}{\eta} \frac{\partial^{2} V_{1}}{\partial z \partial \tau}-\frac{\alpha_{0} C_{0}}{2 C_{\mathrm{b}}} \frac{\partial^{2} V_{1}^{2}}{\partial z^{2}}+\frac{C}{C_{\mathrm{b}}} \frac{\partial^{2}}{\partial z^{2}}\left(V_{1}-W_{1}\right) \\
& -R_{1} C_{\mathrm{b}} \eta \frac{\partial V_{1}}{\partial z}=\frac{1}{12} \frac{\partial^{4} V_{1}}{\partial z^{4}} .
\end{aligned}
$$

By integration of Eqs. (15) and (16) once with respect to $z$, and scaling $W_{1}, V_{1}, \tau$ and $z$ as $W_{1}=\frac{6 \gamma C_{\mathrm{b}}}{\alpha_{0} C_{0}} \psi, V_{1}=-\frac{6 \gamma C_{\mathrm{b}}}{\alpha_{0} C_{0}} \phi$, $\tau=\frac{2}{\eta} T$ and $z=\gamma u$ where $\gamma=1 / \sqrt[3]{12}$, we find:

$\frac{\partial \psi}{\partial T}-6 \psi \frac{\partial \psi}{\partial u}+\frac{\partial^{3} \psi}{\partial u^{3}}=P_{1}(u, T)$,

$\frac{\partial \phi}{\partial T}-6 \phi \frac{\partial \phi}{\partial u}+\frac{\partial^{3} \phi}{\partial u^{3}}=P_{2}(u, T)$

This last set describes two coupled KdV equations, in which the quantities $P_{i}(u, T), i=1,2$ grouping the coupling and the resistive terms play roles of perturbations and are defined as:

$P_{1}(u, T)=\frac{C}{\gamma C_{\mathrm{b}}} \frac{\partial}{\partial u}(\psi+\phi)-R_{1} C_{\mathrm{b}} \eta \psi$,

$P_{2}(u, T)=\frac{C}{\gamma C_{\mathrm{b}}} \frac{\partial}{\partial u}(\phi+\psi)-R_{1} C_{\mathrm{b}} \eta \phi$.

When $P_{i}(u, T)=0$, the two homogeneous KdV equations $\partial_{T} \psi-6 \psi \partial_{u} \psi+\partial_{u}^{3} \psi=0$ and $\partial_{T} \phi+6 \phi \partial_{u} \phi+\partial_{u}^{3} \phi=0$ admit the following one-soliton solutions [5]:

$\psi=-2 \kappa_{1}^{2} \operatorname{sech}^{2} y_{1}$,

$\phi=-2 \kappa_{2}^{2} \operatorname{sech}^{2} y_{2}$ where $y_{1}=\kappa_{1}\left(u-\theta_{1}\right), \theta_{1}=4 \kappa_{1}^{2} T$ and $y_{2}=\kappa_{2}\left(u-\theta_{2}\right)$, $\theta_{2}=4 \kappa_{2}^{2} T$. In the original coordinates, the above onesoliton solutions can be rewritten as:

$$
\begin{aligned}
W(x, t)= & -\frac{3\left(V_{\jmath}+m V_{\mathrm{b}}\right) D_{1}}{\sqrt[3]{12} m} \\
& \operatorname{sech}^{2}\left(\sqrt{\frac{D_{1}}{4 \gamma^{2}}}\left(x-\eta\left(1+\frac{1}{2} \gamma D_{1}\right) t\right)\right), \\
V(x, t)= & \frac{3\left(V_{\mathrm{J}}+m V_{\mathrm{b}}\right) D_{2}}{\sqrt[3]{12} m} \\
& \operatorname{sech}^{2}\left(\sqrt{\frac{D_{2}}{4 \gamma^{2}}}\left(x-\eta\left(1+\frac{1}{2} \gamma D_{2}\right) t\right)\right)
\end{aligned}
$$

with $D_{1}=4 \epsilon \kappa_{1}^{2}$ and $D_{2}=4 \epsilon \kappa_{2}^{2}$. Within the framework of the adiabatic perturbation theory [39], the temporal evolutions of the amplitudes $\kappa_{i}$ and phases $\theta_{i}(i=1,2)$ of the two pulses are determined by solving the following coupled first-order ordinary differential equations:

$\frac{\mathrm{d} \kappa_{i}}{\mathrm{~d} T}=-\frac{1}{4 \kappa_{i}} \int_{-\infty}^{\infty} P_{i}\left(y_{i}, T\right) \operatorname{sech}^{2} y_{i} \mathrm{~d} y_{i}$,

$$
\begin{aligned}
\frac{\mathrm{d} \theta_{i}}{\mathrm{~d} T}= & 4 \kappa_{i}^{2}-\frac{1}{4 \kappa_{i}^{3}} \\
& \int_{-\infty}^{\infty} P_{i}\left(y_{i}, T\right)\left[y_{i}+\frac{1}{2} \sinh \left(2 y_{i}\right)\right] \operatorname{sech}^{2} y_{i} \mathrm{~d} y_{i} .
\end{aligned}
$$

To find explicit forms of these variational equations, we must substitute the pulse solutions (23) and (24) into the perturbation parameters $P_{i}(u, T)$. Doing so, Eqs. (25) and (26) burst into the following four coupled first-order ordinary differential equations:

$$
\begin{aligned}
\frac{\mathrm{d} \kappa_{1}}{\mathrm{~d} T}= & -\frac{8 M}{15} \kappa_{2}^{2} \\
& \operatorname{sech}^{2} A\left[\tanh A+\tanh ^{3} A\right]-\frac{2}{3} N \kappa_{1}, \\
\frac{\mathrm{d} \kappa_{2}}{\mathrm{~d} T}= & \frac{8 M}{15} \kappa_{1}^{2} \\
& \operatorname{sech}^{2} A\left[\tanh A+\tanh ^{3} A\right]-\frac{2}{3} N \kappa_{2},
\end{aligned}
$$

$$
\begin{aligned}
\frac{\mathrm{d} \theta_{1}}{\mathrm{~d} T}= & 4 \kappa_{1}^{2}-M-\frac{M \kappa_{2}^{3}}{\kappa_{1}^{3}} \\
& \operatorname{sech}^{2} A\left[1-\frac{17}{9} \tanh ^{2} A\right],
\end{aligned}
$$




$$
\begin{aligned}
\frac{\mathrm{d} \theta_{2}}{\mathrm{~d} T}= & 4 \kappa_{2}^{2}-M-\frac{M \kappa_{1}^{3}}{\kappa_{2}^{3}} \\
& \operatorname{sech}^{2} A\left[1-\frac{17}{9} \tanh ^{2} A\right],
\end{aligned}
$$

where $M=\frac{C}{\gamma C_{\mathrm{b}}}, N=R_{1} C_{\mathrm{b}} \eta$ and $A=\kappa \Delta \theta$, with $\Delta \theta=\theta_{1}-\theta_{2}$ the phase difference. Leapfrogging of the two interacting solitons corresponds to small oscillations of their amplitudes $\kappa_{i}$ around a common average value $\kappa$. Consequently, we can introduce a small deviation $\lambda_{i}$ from the average amplitude $\kappa$, in such a way that we can write $\kappa_{i}=\kappa+\lambda_{i}$. Similarly the phase difference $\Delta \theta$ must be small. Using the approximation $y_{2} \approx y_{1}+A$ and linearizing Eqs. (27)-(30) become:

$\frac{\mathrm{d} \lambda_{1}}{\mathrm{~d} T}=-\frac{8 M}{15} \kappa^{3} \Delta \theta-\frac{2 N}{3}\left(\kappa+\lambda_{1}\right)$,

$\frac{\mathrm{d} \lambda_{2}}{\mathrm{~d} T}=\frac{8 M}{15} \kappa^{3} \Delta \theta-\frac{2 N}{3}\left(\kappa+\lambda_{2}\right)$,

$\frac{\mathrm{d} \theta_{1}}{\mathrm{~d} T}=4\left(\kappa^{2}+2 \kappa \lambda_{1}\right)-M\left[2-\frac{3}{\kappa} \Delta \lambda\right]$

$\frac{\mathrm{d} \theta_{2}}{\mathrm{~d} T}=4\left(\kappa^{2}+2 \kappa \lambda_{2}\right)-M\left[2+\frac{3}{\kappa} \Delta \lambda\right]$,

with $\Delta \lambda=\lambda_{1}-\lambda_{2}=\kappa_{1}-\kappa_{2}$. From these equations, one obtains:

$\frac{\mathrm{d}^{2} \Delta \theta}{\mathrm{d} T^{2}}+\gamma_{\mathrm{d}} \frac{\mathrm{d} \Delta \theta}{\mathrm{d} T}+\omega_{0}^{2} \Delta \theta=0$

$\Delta \lambda=\frac{\kappa}{\left(8 \kappa^{2}+6 M\right)} \frac{\mathrm{d} \Delta \theta}{\mathrm{d} T}$.

Equations (35) and (36) are reminiscent of the motion of a damped harmonic oscillator, where the damping coefficient $\gamma_{d}$ and the resonance frequency $\omega_{0}$ are defined as:

$\gamma_{\mathrm{d}}=\frac{2 N}{3}, \omega_{0}^{2}=\frac{16}{15} M \kappa^{2}\left(8 \kappa^{2}+6 M\right)$.

It is remarkable that the damping coefficient $\gamma_{d}$ is a linear function of the intraline resistance $R_{1}$, while the resonance frequency $\omega_{0}$ (or the frequency of undamped harmonic oscillations) is proportional to the coupling capacitance $C$. It is worth noting that the adiabatic perturbation theory is valid only when $C_{m} / C_{\mathrm{b}} \ll V_{\mathrm{s}} / V_{\mathrm{b}}$ where $V_{\mathrm{s}}=12 \epsilon \kappa^{2}\left(V_{\mathrm{J}}+m V_{\mathrm{b}}\right) / \sqrt[3]{12} m$ is the average voltage amplitude of the incident solitons. Given that the coupling capacitance $C$ and the intraline resistance $R_{1}$ should be very small consistently with the spirit of the adiabatic perturbation theory, the leapfrogging frequency can only be increased with an increase in the pulse amplitude $\kappa$. In the next section, we shall carry out numerical simulations on the variational Eqs. (31)-(34), in order to gain a more rich insight onto parameter values for which leapfrogging of the pulse pair is more likely to be favoured.

\subsection{Numerical simulations of leapfrogging for the coupled RLC NLTLs}

In ref. [30], an analysis of soliton leapfrogging in a model of coupled NLTLs similar to Fig. 1, but without intraline resistances, has been carried out. Much recently, we have extended the study to the context of two LC-type NLTLs coupled by a linear capacitance with a linear resistance in its shunt branch. In the present study, we shall explore numerically the influence of the intraline resistance on pulse leapfrogging. In this last purpose, we applied a sixthorder Runge-Kutta scheme [40] on the set of four coupled first-order nonlinear ordinary differential Eqs. (27)-(30). To start, we considered small initial values for $\lambda_{i}$ and $\theta_{i}$ and, in addition, selected very close initial values for $\lambda_{1}$ and $\lambda_{2}$, on the one hand, and $\theta_{1}$ and $\theta_{2}$, on the other hand, which are relevant conditions for leapfrogging to occur. Later on, we shall look at the effects of increasing the initial phase and amplitude differences, on the leapfrogging motion.

Graphs in Fig. 2 show time evolutions of $\lambda_{1}$ and $\lambda_{2}$ (left column) and of the amplitude difference $\Delta \lambda$ (right column), for fixed values of the average amplitude $\kappa$ and the capacitive coefficient $M$, but four distinct values of the resistive coefficient $N$, listed in the figure caption.

According to Fig. 2, time evolutions of $\lambda_{1}$ and $\lambda_{2}$ are harmonic oscillations with amplitudes which are more and more exponentially damped with increase in the resistive coefficient $N$. The amplitude difference $\Delta \lambda$ too oscillates harmonically in time, reflecting leapfrogging of the soliton pair. Figures 3, 4 and 5 show the numerical results obtained when the differences in the initial values of the two solitons' amplitudes and phases are increased. One sees that when the differences in the initial values of these parameters increase, their variations are more and more dominated by anharmonic oscillations. Figure 5 shows a total suppression of leapfrogging when the differences between the initial amplitudes and phases become relatively large.

\section{Analysis of leapfrogging for coupled LC NLTLs with impurity}

Let us now consider two coupled NLTLs without intraline resistance as represented in Fig. 6. This model was studied in ref. [30], with an emphasis on conditions under which soliton leapfrogging is expected. 

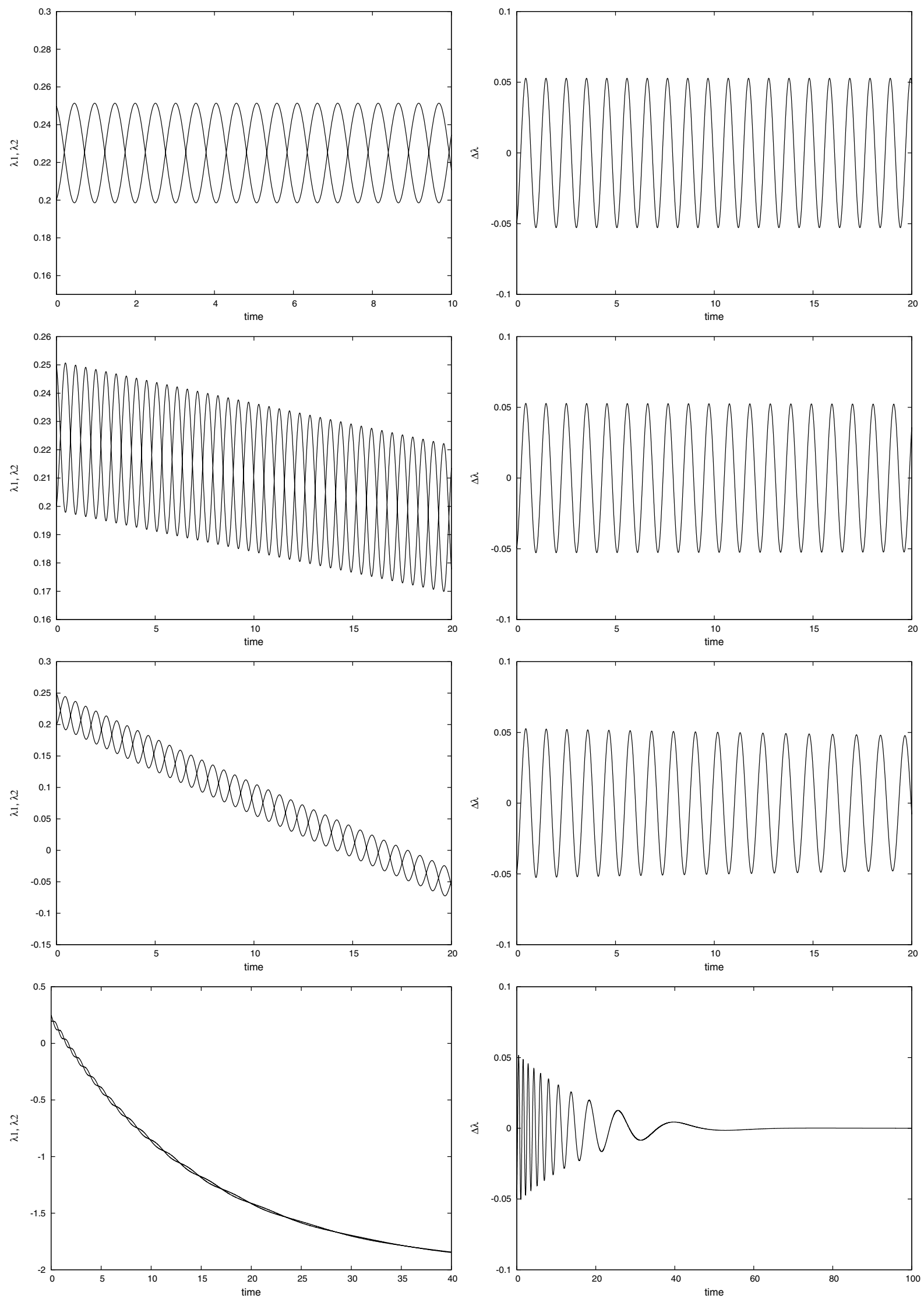
4 Fig. 2 Left column: time variations of $\lambda_{1}$ (downward from $T=0$ ) and $\lambda_{2}$ (upward from $T=0$ ), right column: time variations of $\Delta \lambda$, the initial values are $\lambda_{1}=0.25, \lambda_{2}=0.2, \theta_{1}=0.25, \theta_{2}=0.2$. Parameter values are $\kappa=2$ and $M=0.2$. From top to bottom rows: $N=0$, $0.001,0.01,0.1$

Here, we are interested in the evolution of leapfrogging solitons in the electrical transmission network in Fig. 6, when one Schottky diode in line 1 is imperfect. Mathematically, we model the imperfection by an impurity in the feedback part of the diode localized at position $x=x_{0}$ on the NLTL 1. Using a $\delta$ function to represent the localized impurity, the capacitances of the two varactors can be expressed:

$$
\begin{aligned}
& C_{1}(W)=C_{0} /\left[1-\frac{W}{V_{\mathrm{J}}}\left(1-\beta \delta\left(x-x_{0}\right)\right]^{m}\right. \\
& C_{2}(V)=C_{0} /\left(1+\frac{V}{V_{\mathrm{J}}}\right)^{m},
\end{aligned}
$$

where the impurity rate $\beta$ is assumed positive $(0 \leq \beta \leq 1)$. For convenience, we have set the bias voltage $V_{\mathrm{b}}$ to zero and hence $C_{b}=C_{0}$. Also let $\alpha_{1}=\frac{\alpha_{0}}{2}\left(1-\beta \delta\left(x-x_{0}\right)\right)$. The transmission line equations are then given by:

$L \frac{\mathrm{d}}{\mathrm{d} t}\left(J_{n-1}-J n\right)=W_{n-1}-2 W_{n}+W_{n+1}$,

$L \frac{\mathrm{d}}{\mathrm{d} t}\left(I_{n-1}-\ln \right)=V_{n-1}-2 V_{n}+V_{n+1}$,

$J_{n-1}-J n=\frac{\mathrm{d} Q_{n}}{\mathrm{~d} t}+C_{m} \frac{\mathrm{d}}{\mathrm{d} t}\left(W_{n}-V_{n}\right)$

$I_{n-1}-\ln =\frac{d q_{n}}{d t}+C_{m} \frac{\mathrm{d}}{\mathrm{d} t}\left(V_{n}-W_{n}\right)$

In the continuum limit, the right-hand side of Eqs. (40) and (41) are approximated with partial derivatives with respect to $x$. Again from the definition $\mathrm{d} Q_{n}=C\left(W_{n}\right) \mathrm{d} W_{n}$ and $\mathrm{d} q_{n}=C\left(V_{n}\right) \mathrm{d} V_{n}$, Eqs. (40) to (43) reduce to:

$$
\begin{aligned}
\mathrm{LC}_{0} & \left(\frac{\partial^{2} W}{\partial t^{2}}+\alpha_{1} \frac{\partial^{2} W^{2}}{\partial t^{2}}\right)+\mathrm{LC}_{m} \frac{\partial^{2}}{\partial t^{2}}(W-V) \\
= & \frac{\partial^{2} W}{\partial x^{2}}+\frac{1}{12} \frac{\partial^{4} W}{\partial x^{4}} \\
\mathrm{LC}_{0} & \left(\frac{\partial^{2} V}{\partial t^{2}}-\frac{\alpha_{0}}{2} \frac{\partial^{2} V^{2}}{\partial t^{2}}\right)+\mathrm{LC}_{m} \frac{\partial^{2}}{\partial t^{2}}(V-W) \\
= & \frac{\partial^{2} V}{\partial x^{2}}+\frac{1}{12} \frac{\partial^{4} V}{\partial x^{4}}
\end{aligned}
$$

$W(x, t)=\sum_{i=1}^{n} \epsilon^{i} W_{i}(x, t)$,

$V(x, t)=\sum_{i=1}^{n} \epsilon^{i} V_{i}(x, t)$

In addition to transformations introduced in the previous section, we apply the following transformation on the space coordinate:

$z=\epsilon^{\frac{1}{2}}\left(x-\eta_{o} t\right)$

where $\eta_{0}=\left(\mathrm{LC}_{0}\right)^{-1 / 2}$. Substituting Eqs. (46) to (48) into Eqs. (44) and (45) and integrating once with respect to $z$, we obtain the following equations to the order $0\left(\epsilon^{3}\right)$ :

$$
\begin{aligned}
& \frac{2}{\eta} \frac{\partial W_{1}}{\partial \tau}-\alpha_{0} W_{1} \frac{\partial W_{1}}{\partial z}+\frac{1}{12} \frac{\partial^{3} W_{1}}{\partial z^{3}}=\frac{C}{C_{0}} \frac{\partial}{\partial z}\left(W_{1}-V_{1}\right) \\
& \quad-\frac{\alpha_{o} \beta}{2} \int \delta\left(z-z_{0}\right) \frac{\partial^{2} W_{1}^{2}}{\partial z^{2}} \mathrm{~d} z
\end{aligned}
$$

$\frac{2}{\eta} \frac{\partial V_{1}}{\partial \tau}+\alpha_{0} V_{1} \frac{\partial V_{1}}{\partial z}+\frac{1}{12} \frac{\partial^{3} V_{1}}{\partial z^{3}}=\frac{C}{C_{0}} \frac{\partial}{\partial z}\left(V_{1}-W_{1}\right)$

By scaling $W_{1}, V_{1}, \tau$ and $z$ as $W_{1}=\frac{6 \gamma}{\alpha_{0}} \psi, V_{1}=-\frac{6 \gamma}{\alpha_{0}} \phi, \tau=\frac{2}{\eta_{0}} T$ and $z=\gamma u$ where $\gamma$ is the same as defined in the previous section (i.e., $\gamma=1 / \sqrt[3]{12}$ ), we find:

$\frac{\partial \psi}{\partial T}-6 \psi \frac{\partial \psi}{\partial u}+\frac{\partial^{3} \psi}{\partial u^{3}}=P_{3}(u, T)$

$\frac{\partial \phi}{\partial T}-6 \phi \frac{\partial \phi}{\partial u}+\frac{\partial^{3} \phi}{\partial u^{3}}=P_{4}(u, T)$.

The perturbation terms $P_{i}(u, T), i=3,4$ in the present case are given by:

$P_{3}(u, T)=\frac{C}{\gamma C_{0}} \frac{\partial}{\partial u}(\psi+\phi)-\frac{3 \beta}{\gamma} \int \delta\left(u-u_{0}\right) \frac{\partial^{2} \psi_{1}^{2}}{\partial u^{2}} d u$,

$P_{4}(u, T)=\frac{C}{\gamma C_{0}} \frac{\partial}{\partial u}(\phi+\psi)$.

In the absence of perturbations, Eqs. (51) and (52) are two independent $\mathrm{KdV}$ equations admitting one-soliton solutions similar to (21) and (22). With these solutions, the adiabatic perturbation theory leads to the following set of four coupled first-order ordinary differential equations for the variational parameters $\kappa_{i}$ and $\theta_{i}(i=1,2)$ :

We expand the voltage variables $W$ and $V$ in series, i.e., 

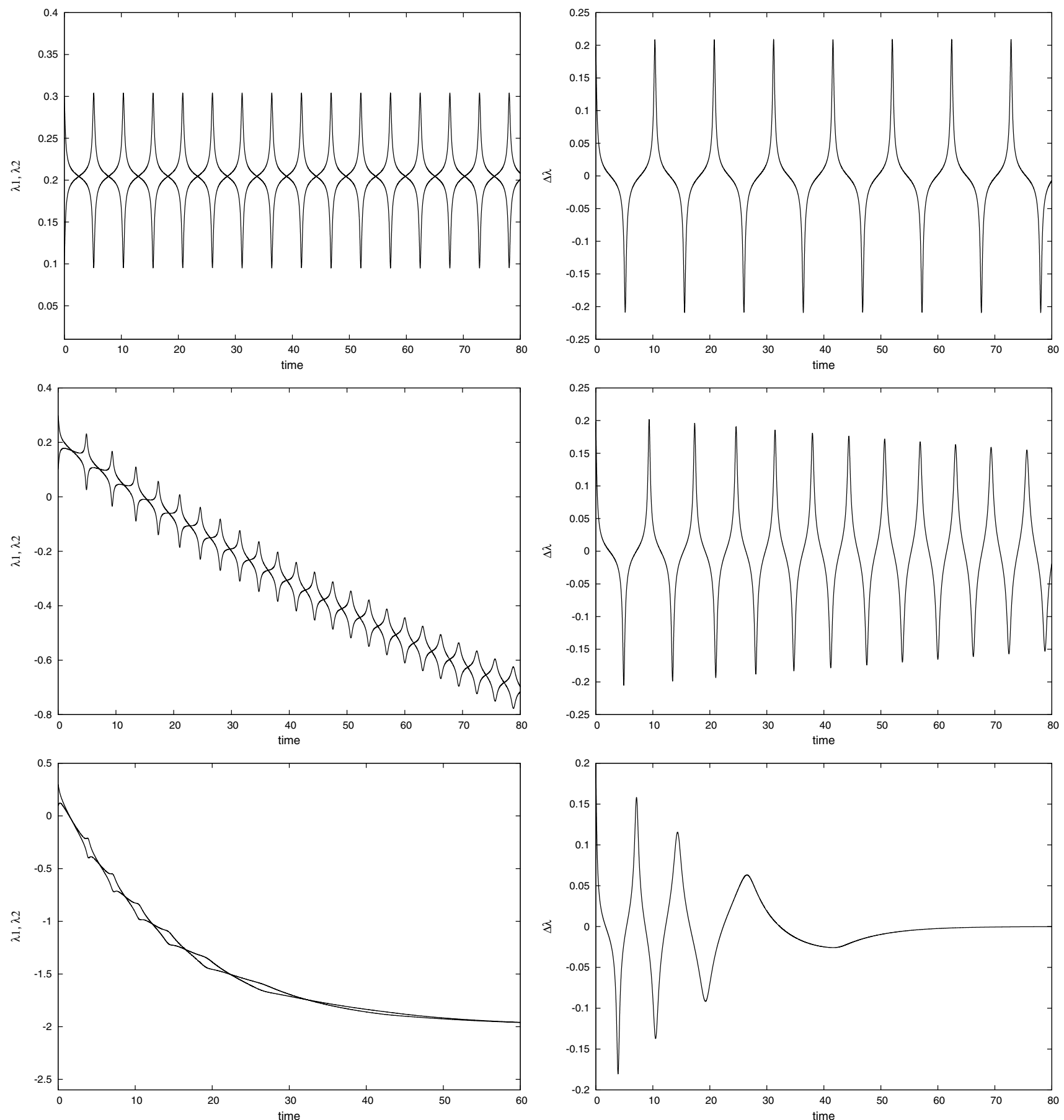

Fig. 3 Left column: time variations of $\lambda_{1}$ (downward from $T=0$ ) and $\lambda_{2}$ (upward from $T=0$ ), right column: time variations of $\Delta \lambda$, the initial values are $\lambda_{1}=0.3, \lambda_{2}=0.1, \theta_{1}=0.28, \theta_{2}=0.1$. Parameter values are $\kappa=2$ and $M=0.2$. From top to bottom rows: $N=0,0.01,0.1$

$$
\begin{aligned}
\frac{\mathrm{d} \kappa_{1}}{\mathrm{~d} T}= & -\frac{8 M_{1} \kappa_{2}^{2}}{15} \operatorname{sech}^{2} A\left[\tanh A+\tanh ^{3} A\right] \\
& +N_{1} \kappa_{1}^{5}\left[4 \operatorname{sech}^{4} y_{0}-5 \operatorname{sech}^{6} y_{0}\right]
\end{aligned}
$$

$$
\frac{\mathrm{d} \kappa_{2}}{\mathrm{~d} T}=\frac{8 M_{1} \kappa_{1}^{2}}{15} \operatorname{sech}^{2} A\left[\tanh A+\tanh ^{3} A\right]
$$

$$
\frac{\mathrm{d} \theta_{1}}{\mathrm{~d} T}=4 \kappa_{1}^{2}-M_{1}-\frac{M_{1} \kappa_{2}^{3}}{\kappa_{1}^{3}} \operatorname{sech}^{2} A\left[1-\frac{17}{9} \tanh ^{2} A\right],
$$



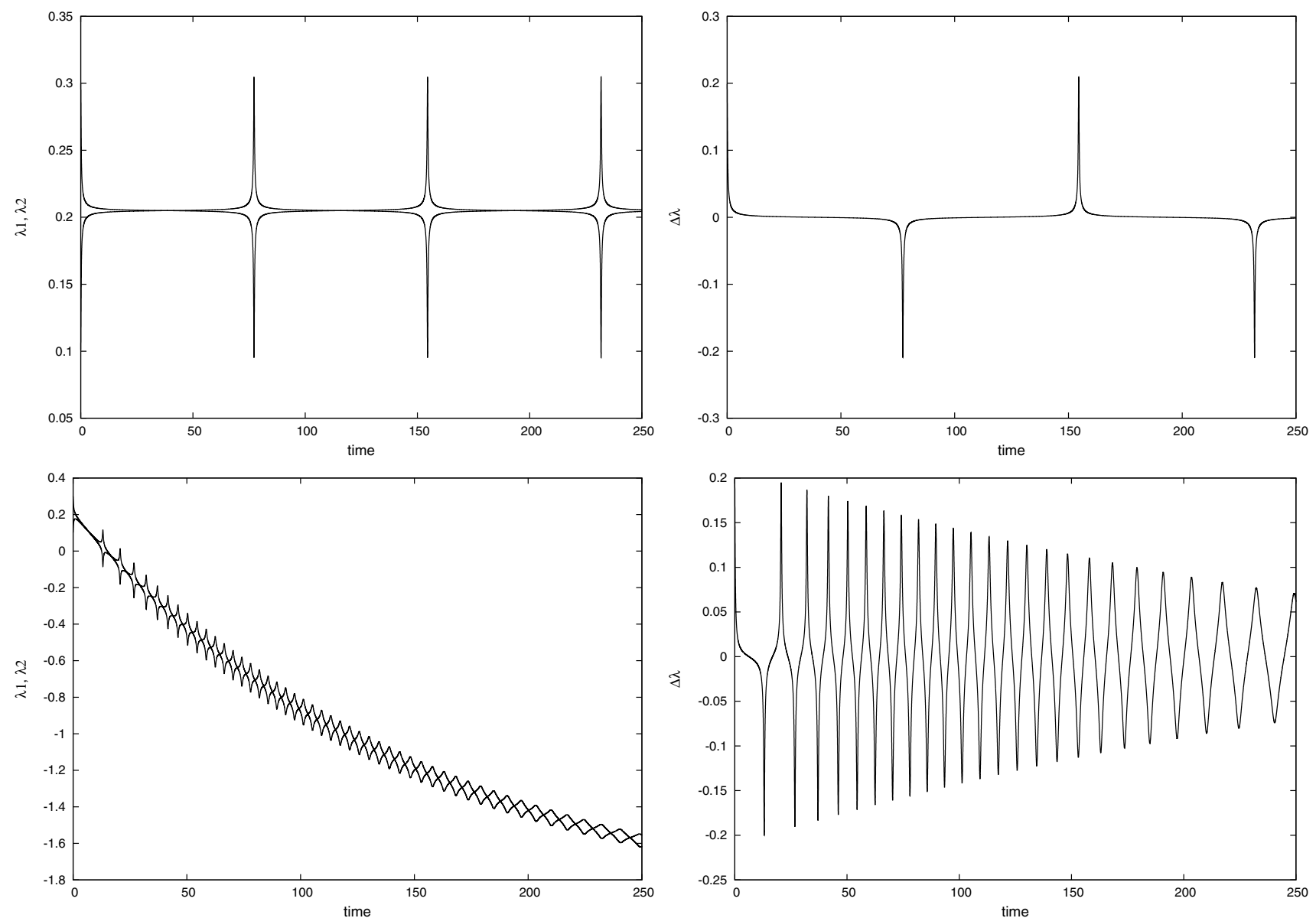

Fig. 4 Left column: time variations of $\lambda_{1}$ (downward from $T=0$ ) and $\lambda_{2}$ (upward from $T=0$ ), right column: time variations of $\Delta \lambda$, the initial values are $\lambda_{1}=0.30076, \lambda_{2}=0.1, \theta_{1}=0.28, \theta_{2}=0.1$. Parameter values are $\kappa=2$ and $M=0.2$ From top to bottom rows: $N=0,0.01$
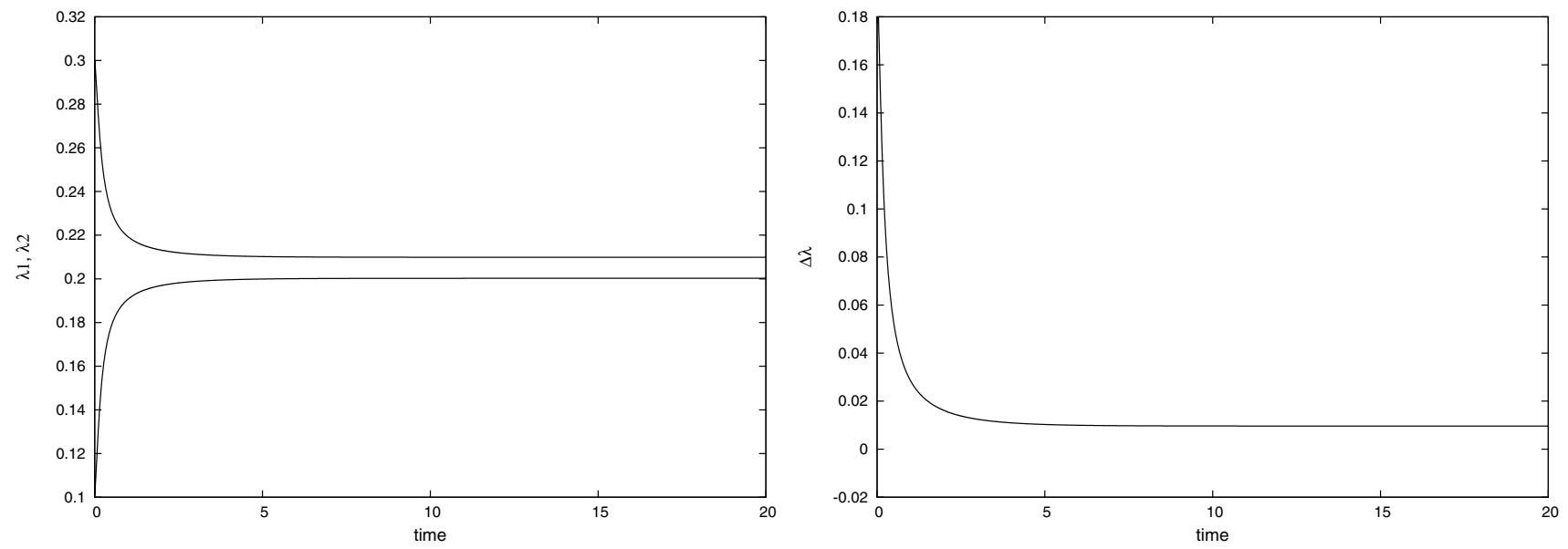

Fig. 5 Left graph: time variations of $\lambda_{1}$ (downward from $T=0$ ) and $\lambda_{2}$ (upward from $T=0$ ), right graph: time variations of $\Delta \lambda$, the initial values are $\lambda_{1}=0.301, \lambda_{2}=0.1, \theta_{1}=0.28, \theta_{2}=0.1$. Parameter values are $\kappa=2, M=0.2, N=0$ 
Fig. 6 Equivalent circuit model of coupled NLTLs. The capacitance at the $n$th cell is given by $C\left(V_{n}\right)$

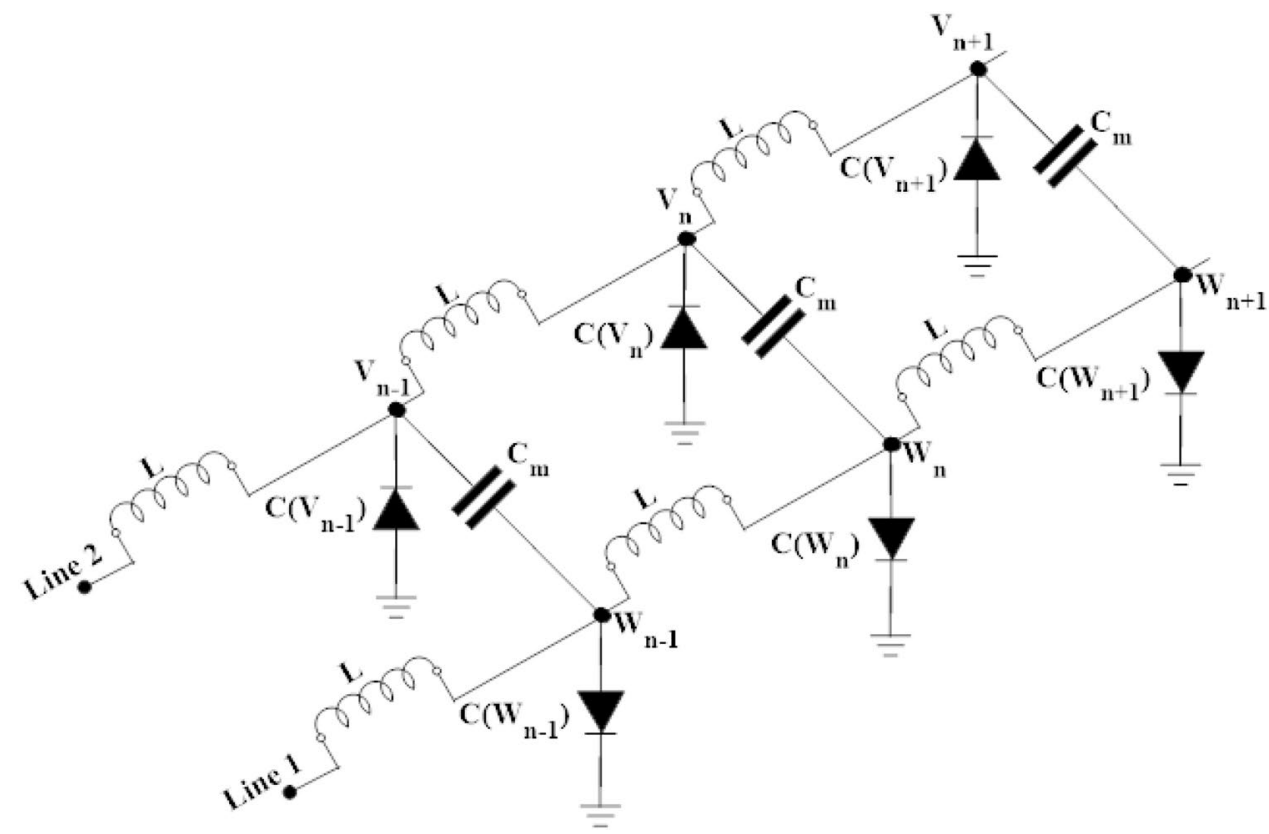

$\frac{\mathrm{d} \theta_{2}}{\mathrm{~d} T}=4 \kappa_{2}^{2}-M_{1}-\frac{M_{1} \kappa_{1}^{3}}{\kappa_{2}^{3}} \operatorname{sech}^{2} A\left[1-\frac{17}{9} \tanh ^{2} A\right]$

where $M_{1}=C / \gamma C_{0}, N_{1}=24 \beta / \gamma$ and $y_{0}=\kappa_{1} u_{0}$. Again we introduce small deviations $\lambda_{i}$ from the average amplitude $\kappa$ of the two solitons as $\lambda_{i}$ as $\kappa_{i}=\kappa+\lambda_{i}$ and the phase difference $\Delta \theta=\theta_{1}-\theta_{2}$ which too must be small. Using the approximation $y_{2} \approx y_{1}+A$, Eqs. (55) to (58) can be linearized and yield:

$$
\begin{aligned}
\frac{\mathrm{d} \lambda_{1}}{\mathrm{~d} T}= & -\frac{8 M_{1}}{15} \kappa^{3} \Delta \theta+\left[\left(49 \kappa^{2} u_{0}-5\right) N_{1} \kappa^{4}\right] \lambda_{1} \\
& +\left(7 \kappa^{2} u_{0}-1\right) N_{1} \kappa^{5} \\
\frac{\mathrm{d} \lambda_{2}}{\mathrm{~d} T}= & \frac{8 M_{1}}{15} \kappa^{3} \Delta \theta
\end{aligned}
$$

$\frac{\mathrm{d} \theta_{1}}{\mathrm{~d} T}=4\left(\kappa^{2}+2 \kappa \lambda_{1}\right)-M_{1}\left[2-3 \kappa^{-1}\left(\lambda_{1}-\lambda_{2}\right)\right]$

$\frac{\mathrm{d} \theta_{2}}{\mathrm{~d} T}=4\left(\kappa^{2}+2 \kappa \lambda_{2}\right)-M_{1}\left[2+3 \kappa^{-1}\left(\lambda_{1}-\lambda_{2}\right)\right]$.

The last two equations suggest that the phase difference $\Delta \theta$ and the amplitude difference $\Delta \lambda$ are related through the differential equation:

$\frac{\mathrm{d} \Delta \theta}{\mathrm{d} T}=\frac{8 \kappa^{2}+6 M_{1}}{\kappa} \Delta \lambda$.

On the other hand, the first two equations lead to the following second-order ordinary differential equation for the amplitude difference: $0=\frac{\mathrm{d}^{2} \Delta \lambda}{\mathrm{d} T^{2}}+\Omega_{0}^{2} \Delta \lambda+F\left(\lambda_{1}, u_{0}\right)$

$\Omega_{0}^{2}=\frac{16 M_{1}}{15} \kappa^{2}\left(8 \kappa^{2}+6 M_{1}\right)$,

$F\left(\lambda_{1}, u_{0}\right)=\left(8 \kappa^{2}+6 M_{1}\right)$

$$
\left[\left(5-49 \kappa^{2} u_{0}\right) N_{1} \kappa^{3} \lambda_{1}+\left(1-7 \kappa^{2} u_{0}\right) N_{1} \kappa^{4}\right] .
$$

Equation (64) is nothing else, but the equation of motion of an harmonic oscillator with the oscillation frequency $\Omega_{0}$, "driven" by a force $F\left(\lambda_{1}, u_{0}\right)$. This drive leads to either an enhancement or a suppression of $\Delta \lambda$.

To be more explicit, the expression of $F\left(\lambda_{1}, u_{0}\right)$ given in formula (65) suggests that the amplitude difference will oscillate with increasing amplitude, when the defective diode is closer to the input end of the transmission line and the average amplitude $\kappa$ of the leapfrogging solitons is not too large. When $\kappa$ is relatively large, the impurity will accelerate the soliton signal on line 1 , thus increasing its speed relative to the speed of soliton on line 2 .

Concerning the issue of the effects of impurities on soliton propagation in NLTLs, it is instructive stressing that the influence of a localized impurity on soliton propagation in NLTLS has been investigated in some past works. It is therefore well established that a default-type impurity will increase the amplitude of a soliton approaching the impurity $[25,26]$, hence causing its acceleration in virtue of the amplitude dependence of the velocity of the $\mathrm{KdV}$ soliton. In recent numerical simulations, Pan et al. [26] obtained that the response of a soliton signal to the 
presence of a localized impurity in an LC NLTL is standard: an excess structural defect will always trap a soliton signal causing its delay, whereas a structural default will accelerate the soliton signal on approaching the impurity, whether the impurity is capacitive or inductive.
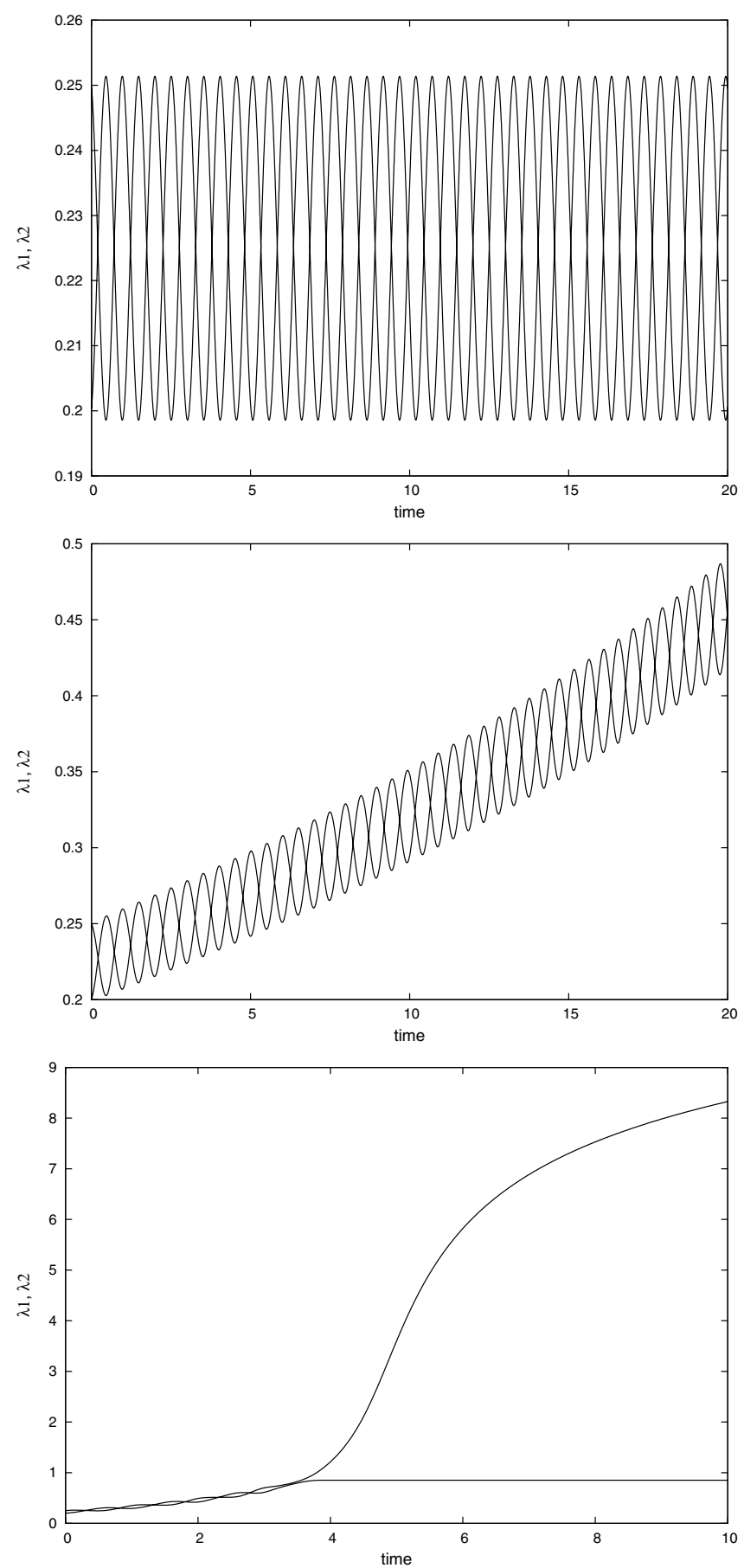

Fig. 7 Left column: time variations of $\lambda_{1}$ (downward from $T=0$ ) and $\lambda_{2}$ (upward from $T=0$ ), right column: time variations of $\Delta \lambda_{\text {, }}$ the initial values are $\lambda_{1}=0.25, \lambda_{2}=0.2, \theta_{1}=0.25, \theta_{2}=0.2$. Param-
We carried out numerical simulations of the set of coupled first-order nonlinear ordinary differential equation (55)-(58), still with a sixth-order Runge-Kutta algorithm with fixed step. The initial values, as well as values of characteristic parameters of the model, are the same we used for Fig. 2 (see values in the caption of Fig. 7). Results are
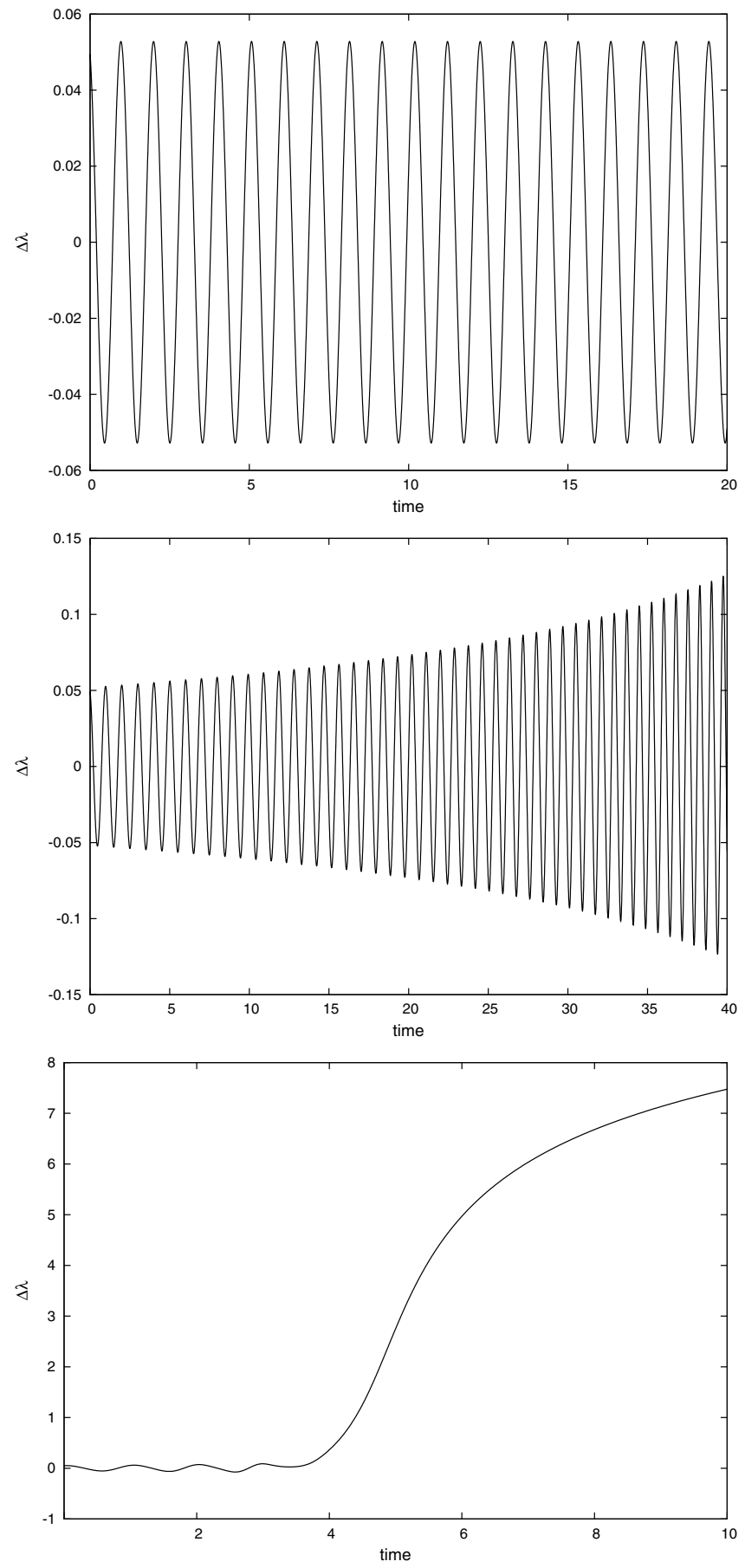

eter values are $\kappa=2, M_{1}=0.2, u_{0}=0.3$. From top to bottom rows: $N_{1}=0,0.001$ and 0.01 
shown in Fig. 7 , where we plotted $\lambda_{1}$ and $\lambda_{2}$, and the amplitude difference, for different values of the impurity coefficient $N_{1}$.

Graphs in Fig. 7 show that the soliton leapfrogging is a regular harmonic oscillation with constant maximum amplitudes when there is no impurity. However, as the impurity rate $\beta$ (an hence the impurity coefficient $N_{1}$ ) is increased from zero, the amplitude difference $\Delta \lambda$ oscillates with the maximum oscillation amplitudes increasing with time. When $N_{1}$ attains a critical value, the leapfrogging is suppressed after a short time. A look at the variations of $\lambda_{1}$ and $\lambda_{2}$ with time on the left graph clearly suggests that $\lambda_{1}$ gets amplified after a short propagation time, but not $\lambda_{2}$, implying an acceleration of the soliton signal on line 1 relative to its counterpart on line 2 .

\section{Conclusion}

We have investigated the leapfrogging dynamics of a pair of $\mathrm{KdV}$ solitons in two nonlinear transmission lines, weakly coupled by a linear capacitance. Two different physical configurations of coupled nonlinear transmission lines were considered: the first model was two RLC lines with intraline Schottky varactors, and in the second model, we considered two coupled LC lines one of which had a localized capacitive impurity. For the first model, we obtained that adding the resistive element along with the feedback capacitor on the coupled transmission lines causes a damping of the soliton amplitudes, thus acting against leapfrogging. For leapfrogging to survive the presence of the resistive component, the average amplitude of the two interacting solitons should be large enough and the resistance relatively small, consistently with the spirit of the adiabatic perturbation theory. For the second model, we established that a defect in one of the Schottky diodes on line 1 with accelerate the soliton signal on the line, causing a drive of the second soliton with the possibility of their leapfrogging as long as the impurity rate is relatively small. As we increase the impurity rate, the soliton signal in line 1 gains in amplitude and consequency in speed, and hence cannot be followed by the soliton signal in line 2 . In this case, no leapfrogging can occur.

The effects of a localized impurity on soliton signals in NLTLs have been investigated in several previous works; it is there well established that a localized impurity will always accelerate a soliton approaching the impurity when it is a structural default in the defective electrical component $[25,26]$. This response of KdV soliton to the presence of a localized impurity in the NLTL is actually universal; indeed, similar behaviours are predicted in many other distinct physical systems such as Josephson junction transmission lines [44, 45], Frenkel-Kontorova systems [46, 47] and double-well systems [48].

Acknowledgements A. M. Dikandé wishes to acknowledge support from the Alexander von Humboldt foundation. He also thanks Pr. Holger Kantz at MPIPKS Dresden for hosting his visit in the "Nonlinear Time Series Analysis" research group.

\section{Compliance with ethical standards}

Conflict of interest The authors declare that they have no conflict of interest.

Open Access This article is distributed under the terms of the Creative Commons Attribution 4.0 International License (http://creativecommons.org/licenses/by/4.0/), which permits unrestricted use, distribution, and reproduction in any medium, provided you give appropriate credit to the original author(s) and the source, provide a link to the Creative Commons license, and indicate if changes were made.

\section{References}

1. Hirota R, Suzuki K (1970) Studies on lattice solitons by using electrical networks. J Phys Soc Jpn 28:1366

2. Jager D (1985) Characteristics of travelling waves along the nonlinear transmission lines for monolithic integrated circuits: a review. Int J Electron 58:649

3. Toda M (1967) Vibration of a chain with nonlinear interaction. J Phys Soc Jpn 22:431

4. Toda M (1989) Theory of nonlinear lattices, 2 enl edn. Springer, Berlin

5. Ablowitz M, Clarkson P (1991) Solitons, nonlinear evolutions and inverse scattering. Cambridge University Press, Cambridge

6. Drazin PG, Johnson RS (1996) Solitons: an introduction. Cambridge University Press, Cambridge

7. Filippov AT (2010) Versatile soliton. Birkhäuser, Basel

8. Ricketts DS, Ham D (2011) Electrical solitons: theory, designs and applications. CRC Press, Boca Raton

9. TaoT (2009) Why are solitons stable? Bull AMS 46:33

10. Kengne $E$, Vaillancourt $R$ (2009) Propagation of solitary waves on lossy nonlinear transmission lines. Int J Mod Phys B 23:1

11. Afshari $E$, Hajimiri $A$ (2005) Nonlinear transmission lines for pulse shaping in silicon. IEEE J Sol State Circuits 40:744

12. Kengne E, Liu WM (2006) Exact solutions of the derivative nonlinear Schrödinger equation for a nonlinear transmission line. Phy Rev E 73:0266031

13. Ndzana Fll, Mohamadou A, Kofané TC, English LQ (2008) Modulated waves and pattern formation in coupled discrete nonlinear LC transmission lines. Phys Rev E 78:016606

14. Ndzana FII, Mohamadou A, Kofané TC (2008) Modulational instability in a purely nonlinear coupled complex Ginzburg-Landau equations through a nonlinear discrete transmission line. Chaos 18:043121

15. Narahara K (2003) Traveling-wave retimer with coupled nonlinear transmission line. Jpn J Appl Phys 42:1192

16. Kuusela T, Hietarinta J, Kokko K, Laiho R (1987) Soliton experiments in a nonlinear electrical transmission line. Eur J Phys 8:27

17. Kuusela T (1995) Soliton experiments in transmission lines. Chaos Solitons Fractals 5:2419

18. Essimbi BZ, Dikandé AM, Kofané TC, Zibi AA (1995) Asymmetric gap solitons in a non-linear LC transmission line. Phys Scrip $52: 17$ 
19. Essimbi BZ, Dikandé AM, Kofané TC, Zibi AA (1995) Localized solitary signals on a coupled nonlinear transmission line. J Phys Soc Jpn 64:2777

20. Singer AC, Oppenheim AV, Wornell GW (1999) Detection and estimation of multiplexed soliton signals. IEEE Trans Sign Proc 47:2768

21. Dikandé AM, Ga-Akeku B (2009) Localized short impulses in a nerve model with self-excitable membrane. Phys Rev $E$ 80:041904

22. Oriols X, Martin F (2001) Analytical solitons in nonlinear transmission lines loaded with heterostructure barrier varactors. $J$ Appl Phys 90:2595

23. Sato M, Yasui S, Kimura M, Hikihara T, Sievers AJ (2007) Management of localized energy in discrete nonlinear transmission lines. Euro Phys Lett 80:30002

24. Tsuboi T, Toyama FM (1991) Computer experiments on solitons in a nonlinear transmission line I. Formation of stable solitons. Phys Rev A 44:2686

25. Tsuboi T, Toyama FM (1991) Computer experiments on solitons in a nonlinear transmission line II. Propagation of solitons in an impurity-doped line. Phys Rev A 44:2691

26. Pan JT, Chen WZ, Tao F, Xu W (2011) Influence of impurities on solitons in the nonlinear LC transmission line. Phys Rev $E$ 83:016601

27. Jäger D (1982) Experiments on KdV solitons. J Phys Soc Jpn $51: 1686$

28. Gasch A, Berning T, Jäger D (1986) Generation and parametric amplification of solitons in a nonlinear resonator with a Korteweg-de Vries medium. Phys Rev A 34:4528

29. Narahara K (2010) Characterization of nonlinear transmission lines for short pulse amplification. J Infrared Millim Terahertz Waves 31:411

30. Narahara K (2015) Characterization of leapfrogging solitary waves in coupled nonlinear transmission lines. Nonlinear Dyn $81: 1805$

31. Nkongho Achere A, Akong Ngate L, Dikandé AM, Essimbi BZ (2019) Leapfrogging dynamics of interacting solitons in weakly coupled nonlinear transmission lines. SN Appl Sci 1:552

32. Hietala N, Hänninen R, Salman H, Barenghi CF (2016) Leapfrogging Kelvin waves. Phys Rev Fluids 1:084505

33. Lui AK, Kubota T, Ko DRS (1980) Resonant transfer of energy between nonlinear waves in neighbouring pycnoclines. Stud Appl Math 63:26
34. Lui AK, Pereira NR, Ko DRS (1982) Weakly interacting internal solitary waves in neighbouring pycnoclines. J Fluid Mech 122:187

35. Weidman PD, Johnson M (1982) Experiments on leapfrogging internal solitary waves. J Fluid Mech 122:195

36. Gear JA, Grimshaw R (1984) Weak and strong interactions between internal solitary waves. Stud Appl Math 70:235

37. Nitsche $M$, Weidman PD, Grimshaw R, Ghrist M, Fornberg B (2010) Evolution of solitary waves in a two-pycnocline system. J Fluid Mech 642:235

38. Malomed BA (1987) Leapfrogging solitons in a system of coupled KdV equations. Wave Motion 9:401

39. Kivshar YS, Malomed BA (1989) Dynamics of solitons in nearly integrable systems. Rev Mod phys 61:763

40. Luther HA (1968) An explicit sixth-order Runge-Kutta formula. Math Comput 22:434

41. Jie H, Qian Z, Hao Y, Junrong D, Haiying Z (2014) Planar Schottky varactor diode and corresponding large signal model for millimeter-wave applications. J Semicond 35:0540061

42. Boylestad R, Nashelsky L (2013) Electronic devices and circuit theory, 7th edn. Prentice Hall, Englewood Cliffs

43. Jeffrey A, Kawahara T (1982) Asymptotic methods in nonlinear wave theory. Pitman, London

44. Shnirman A, Ben-Jacob E, Malomed BA (1997) Tunneling and resonant tunneling of fluxons in a long Josephson junction. Phy Rev B 55:14677

45. Starodub IO, Zolotaryuk Y (2012) Scattering of quasi-one-dimensional solitons on impurities in large Josephson junctions. Phys Lett A 376:3101

46. Malomed BA (1988) Interaction of a soliton with an impurity in the sine-Gordon model of a commensurate charge-densitywave system. J Phys C 21:5163

47. Braun OM, Kivshar YS (1998) Nonlinear dynamics of the FrenkelKontorova model. Phys Rep 306:1

48. Dikandé AM, Kofané TC (1994) Oscillatory motions of solitons in finite inhomogeneous structures. J Phys Condens Matter 6:6229

Publisher's Note Springer Nature remains neutral with regard to jurisdictional claims in published maps and institutional affiliations. 